\title{
Pre-earthquake magnetic pulses
}

\author{
J. Scoville ${ }^{1,2,3}$, J. Heraud ${ }^{4}$, and F. Freund ${ }^{1,2,3}$ \\ ${ }^{1}$ San Jose State University, Dept. of Physics, San Jose, CA 95192-0106, USA \\ ${ }^{2}$ SETI Institute, Mountain View, CA 94043, USA \\ ${ }^{3}$ NASA Ames Research Center, Moffett Field, CA 94035, USA \\ ${ }^{4}$ Pontificia Universidad Católica del Perú, Lima, Peru
}

Correspondence to: J. Scoville (atpsynthase@mail.com)

Received: 22 September 2014 - Accepted: 19 November 2014 - Published: 20 August 2015

\begin{abstract}
A semiconductor model of rocks is shown to describe unipolar magnetic pulses, a phenomenon that has been observed prior to earthquakes. These pulses are suspected to be generated deep in the Earth's crust, in and around the hypocentral volume, days or even weeks before earthquakes. Their extremely long wavelength allows them to pass through kilometers of rock. Interestingly, when the sources of these pulses are triangulated, the locations coincide with the epicenters of future earthquakes. We couple a drift-diffusion semiconductor model to a magnetic field in order to describe the electromagnetic effects associated with electrical currents flowing within rocks. The resulting system of equations is solved numerically and it is seen that a volume of rock may act as a diode that produces transient currents when it switches bias. These unidirectional currents are expected to produce transient unipolar magnetic pulses similar in form, amplitude, and duration to those observed before earthquakes, and this suggests that the pulses could be the result of geophysical semiconductor processes.
\end{abstract}

\section{Introduction}

Rocks, especially igneous rocks, behave as semiconductors under conditions of temperature and pressure that occur in the Earth's crust (Selway, 2014; Unsworth et al., 1999; Freund, 2003). Semiconductor behavior has also been demonstrated in laboratory settings using rocks at room temperatures (Freund, 2002, 2010; Freund et al., 2006; Dahlgren et al., 2014; Scoville et al., 2015).

Although the magnetic fields produced by small semiconductors are often negligible, semiconductors on geophysical scales may produce significant magnetic fields. This is of par- ticular interest since these fields can pass through the crust, potentially conveying information about the state of rocks deep below.

Ultra-low ${ }^{1}$ frequency (ULF) electromagnetic emissions are reported as having been observed prior to earthquakes (Bleier et al., 2009), possibly resulting from electric currents flowing deep in the crust (Bortnik et al., 2010). Increased levels of magnetic fluctuations have been repeatedly observed prior to earthquakes since at least 1964 (Moore, 1964), but these transient phenomena are not yet fully understood and their applicability as earthquake precursors remains controversial within the geophysical community as many claimed precursory signals have been disputed or disproven in subsequent studies. For example, one of the most frequently cited magnetic anomalies preceded the Loma Prieta earthquake (Fraser-Smith et al., 1990). Some authors dismiss this as normal geomagnetic activity enhanced by operator or amplifier malfunction (Campbell, 2009; Thomas et al., 2009), while counterarguments (Fraser-Smith et al., 2011) point out that continuous calibration tests should preclude this as a possibility, that the precursor lacks the diurnal behavior of typical geomagnetic activity, and that amplifier malfunction would not preferentially amplify low-frequency signals. (FraserSmith et al., 2011) states that “...there is independent scientific support for us believing that the magnetic field fluctuations may have been precursory, but quite obviously further independent confirming measurements are required, accord-

\footnotetext{
${ }^{1}$ In this context, "ultra-low" refers to electromagnetic waves having frequencies from millihertz to a few Hertz, in contrast to the International Telecommunications Union (ITU) definition of ultra-low, which would correspond to waves having frequencies of $300 \mathrm{~Hz}-3 \mathrm{kHz}$.
} 
ing to the normal scientific process, before such magnetic field changes can be referred to definitively as precursors."

There are also reports of electric signals being associated with seismicity without a significant magnetic component. This is frequently the case with the Varotsos VAN method (Varotsos, 2005), presumably due to electrostatic charge accumulating at the Earth's surface.

During the weeks leading up to the $M=5.4$ Alum Rock earthquake of 30 October 2007, a magnetometer located about $2 \mathrm{~km}$ from the epicenter recorded unusual nonalternating magnetic pulses, reaching amplitudes up to $30 \mathrm{nT}$ (Bortnik et al., 2010; Bleier et al., 2009). The incidence of these pulses increased as the day of the earthquake approached. Figure 4 from Bleier et al. (2009) shows that in the 3 weeks preceding the Alum rock earthquake, the pulse count was much higher than any other 3-week period from 2006-2007. After the earthquake, the pulse count immediately falls back to normal levels. (Bleier et al., 2009) also note that there were no nearby lightning strikes at corresponding times, and that in comparison to the PC 3 and PC4 geomagnetic pulsations, these localized unipolar pulses were much stronger and lasted longer, and could not be seen at locations far from the EQ epicenter, as would be expected for geomagnetic activity. Observed pre-earthquake electromagnetic waves typically have frequencies between 0.01 and $20 \mathrm{~Hz}$, possibly owing to the fact that only low-frequency components may traverse tens of kilometers through the rock column.

A pair of magnetometer stations in Peru recently recorded similar unipolar pulses prior to several medium-sized earthquakes, and triangulating the source of these pulses revealed the location of subsequent earthquake epicenters (Heraud et al., 2013). The unipolar magnetic pulses observed prior to earthquakes have a characteristic shape that can be seen in Fig. 1. The unipolar nature of the magnetic pulses is somewhat unusual and bears resemblance to pulses produced by lightning and other electrical breakdown phenomena. However, the duration of many pre-earthquake pulses exceeds several seconds, much longer than any lightning strike. Moreover, triangulation of such pulses near Lima, Peru revealed that strong pulses originated almost exclusively from locations within a few kilometers of future earthquake epicenters (Heraud et al., 2013).

To model the electromagnetic phenomena associated with volumes of rock, we solve a three-dimensional drift-diffusion model of a semiconductor and calculate the magnetic fields induced by its electric currents. The model is seen to describe transient low-frequency unipolar magnetic pulses.

\section{Rocks as semiconductors}

The conductivity of crustal rocks in fault zones has been measured by magnetotellurics (Selway, 2014; Unsworth et al., 1999) as having conductivity values too low to be a

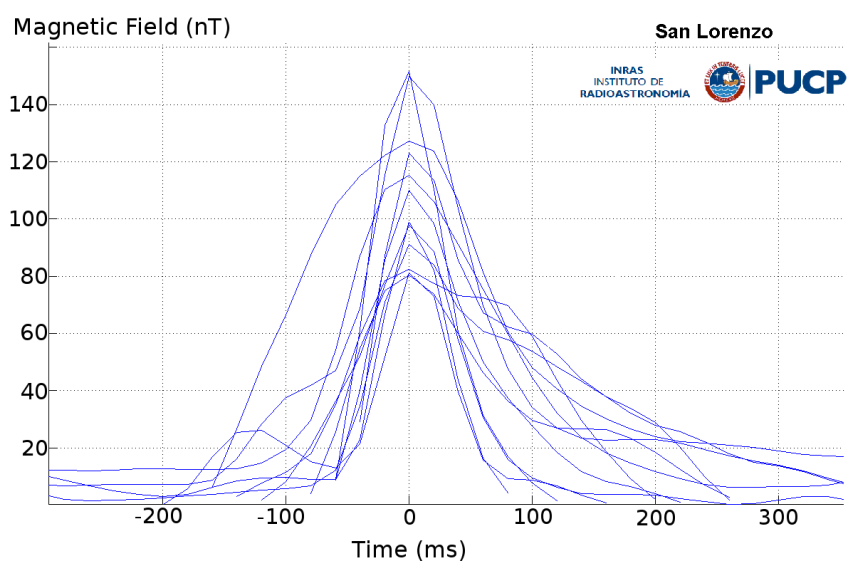

Figure 1. Magnetic pulses observed prior to an earthquake in Lima, Peru, approximately $25 \mathrm{~km}$ from the epicenter.

conductor but too high to be an insulator. Such a material is called a semiconductor (Harris, 1998). Rocks having conductivities in the range $0.1-1 \mathrm{Sm}^{-1}$ have been observed in magnetotelluric surveys, e.g. from deep crustal rocks along the San Andreas fault (Unsworth et al., 1999). Although there is no generally accepted reason for such high levels of conductivity, several hypotheses have been proposed, such as fluid saturation (Unsworth et al., 1999), partial melting (Selway, 2014), intergranular carbon films (Duba and Shankland, 1982), and the presence of mobile charge carriers referred to as positive holes (Freund, 2003).

We will show that unipolar pulses can emerge simply from the electrical drift and random diffusion of charge carriers in a semiconducting volume of rock. There are several reasons why this is a plausible mechanism for the observed pulses. Large electrical currents are known to accompany earthquakes, occasionally so large that luminous effects known as earthquake lights (Thériault et al., 2014) become apparent. There is experimental evidence (Freund, 2002, 2010; Freund et al., 2006; King and Freund, 1984; Scoville et al., 2015) indicating that, during stressing, electrons and holes are freed in igneous rocks and become available to populate states in the conduction and valence bands, respectively.

One proposed source of charge carriers in rock is the break-up of peroxy defects (Freund, 2002, 2010; Freund et al., 2006; Scoville et al., 2015) as a result of the increase in tectonic stresses. The oxygen sublattice of a wide variety of silicate minerals can form peroxy defects that act as sources of electron/hole pairs (Freund, 2010), causing these minerals to exhibit semiconductivity. Once activated, highly mobile electronic charge carriers diffuse through the minerals.

Peroxy defects are point defects, typically introduced through the incorporation of $\mathrm{H}_{2} \mathrm{O}$ into nominally anhydrous minerals that crystallize in $\mathrm{H}_{2} \mathrm{O}$-laden magmas or recrystallize in high-temperature $\mathrm{H}_{2} \mathrm{O}$-laden environments (Freund, 2010). The incorporation of $\mathrm{H}_{2} \mathrm{O}$ into oxides and silicates leads to $\mathrm{OH}^{-}$pairs that subsequently undergo redox conver- 
sion. The two $\mathrm{H}^{+}$of the $\mathrm{OH}^{-}$pairs combine to form $\mathrm{H}_{2}$, and the $\mathrm{O}^{-}$ions bind to form a peroxy bond. The formation of these peroxy bonds has been extensively studied in laboratory experiments (Freund, 2010, 2002; Freund et al., 1991; Griscom, 2011) and treated by computational chemistry (Ricci et al., 2001).

When peroxy bonds are energized via stresses in the rock or by heat, they may produce electron-hole pairs. The peroxy bond breaks, forming a transient state with two unpaired electrons. This is followed by a fully dissociated state in which a hole is free to move through the crystal structure. A neighboring oxygen atom donates an electron and becomes a hole, as its valence shell becomes deficient by one electron. The donated electron becomes trapped near the broken peroxy bond (Griscom, 2011) in a new state whose energy level is slightly below the upper edge of the valence band. In terms of the valence state, the neighboring oxygen atom, which was previously in an $\mathrm{O}^{2-}$ state, becomes $\mathrm{O}^{-}$. This oxygen anion in the 1 -state is effectively a positive hole with an incomplete valence shell and could also be regarded as an unstable oxygen radical (Freund, 2002).

Holes are capable of propagating through the oxygen lattice, exchanging valence electrons by a phonon-assisted vacancy hopping mechanism (Shluger et al., 1992). This process effectively constitutes a diffusion of $\mathrm{O}^{-}$states through a lattice of $\mathrm{O}^{2-}$ atoms. The trapped electrons are immobile but participate through recombination and electrostatic interactions. Positive hole charge carriers propagate at 100$200 \mathrm{~m} \mathrm{~s}^{-1}$ (Freund, 2002; Scoville et al., 2015), much slower than piezoelectric effects or crack propagation, which propagate at the speed of sound $\left(2000-8000 \mathrm{~m} \mathrm{~s}^{-1}\right)$, but faster than ion transport which takes place at much slower speeds. The charge carrier velocity allows mechanisms such as piezoelectricity, crack dislocation effects (Vallianatos et al., 2004), and the electrokinetic effect (Revil et al., 2003) to be ruled out as the predominant source of electrical activity in many rocks.

A recent study (Dahlgren et al., 2014) has suggested that if crustal semiconductivity is the result of fluid-saturation, the positive hole model might not apply. This conclusion was based on a series of experiments involving dry and fluidsaturated rock samples. However, even if the fluid-saturation hypothesis is valid, analysis of these results ${ }^{2}$ as well as the

\footnotetext{
${ }^{2}$ In Dahlgren et al. (2014), the authors conclude that stressstimulated currents do not occur in fluid-saturated samples of gabbro. Figure 4b in Dahlgren et al. (2014), however, clearly indicates that, given the "ST1" experimental setup (which is erroneously cited several times as originating from Freund, 2002) currents change in response to stress. These are, in fact, stronger than those observed in dry samples, as expected due to their increased conductivity. Figure 5b in Dahlgren et al. (2014), based on the "ST2" experimental setup, shows a $43 \%$ increase in current from an unusually high baseline of 4.85 to about $6.95 \mathrm{nA}$ over the course of seven loadingunloading cycles. In spite of these current changes, Dahlgren et al. (2014) conclude that stress-stimulated currents are not observed in fluid-saturated samples of gabbro.
}

experimental results of a more recent study (Scoville et al., 2015) contradict certain results ${ }^{3}$ of Dahlgren et al. (2014). Moreover, the true conditions of rocks at depth are difficult to emulate in a laboratory setting. At depths of more than a few kilometers the pore spaces of rocks are closed by the overload pressure. Without a connected pore space, no contiguous voids exist within rocks for water or other fluids to fill. Furthermore, liquid water cannot exist at any pressure when temperatures exceed $373.95 \mathrm{C}$, which is the case deep in the crust. At the temperatures and pressures found in the deep crust, water exists not as a liquid but as highly reactive supercritical fluid with very different physical and chemical properties and would be consumed by mineral reactions over geologically short timescales.

The dynamics of other charge carriers (polarons) (Selway, 2014) behave in a manner similar to positive holes, via a vacancy-hopping mechanism. For example, electrons can hop between $\mathrm{Fe}^{3+}$ and $\mathrm{Fe}^{2+}$ atoms in a manner similar to $\mathrm{O}^{-}$ and $\mathrm{O}^{2-}$ atoms, albeit with different mobility and diffusion parameters. Regardless of the mechanism by which semiconductivity arises in crustal rocks, semiconductors on macroscopic scales generally obey the same dynamics of drift and diffusion presented in the next section.

(Scoville et al., 2015) reports a series of experiments relevant to the phenomenon of unipolar pulses. By applying a rapid (approx. 1ms) impulse to a rock tile, a predominantly unipolar pulse of current is consistently observed. Since currents act as sources for the magnetic field, the predominantly unipolar current pulses observed in (Scoville et al., 2015) should be accompanied by predominantly unipolar magnetic pulses. This can be seen in Fig. 2, showing the result of five experiments that deliver a rapid (but non-destructive) force to dry gabbro samples. In the following section, we show that the semiconductor model predicts this behavior in response to charge carrier injection.

The broadening of the $\approx 1 \mathrm{~ms}$ impulse to a $3-6 \mathrm{~ms}$ current pulse indicates that diffusion is taking place as the charge carriers drift through the rock, a behavior that is characteristic of semiconductors. This is the phenomenon that originally allowed Haynes and Shockley (Shockley et al., 1949; Shockley, 1964) to confirm the drift-diffusion dynamics of semi-

\footnotetext{
${ }^{3}$ In addition to an opinion on stress-stimulated currents, Dahlgren et al. (2014) conclude that the polarity (negative) of observed currents in dry gabbro was opposite to that previously reported in the literature (positive). Some of the experiments reported in Scoville et al. (2015) reproduce the ST1 setup reported in Dahlgren et al. (2014) for dry gabbro, obtaining a positive polarity that agrees with the existing literature and contradicts the conclusion of Dahlgren et al. (2014). The anomalous results of Dahlgren et al. (2014) might be explained by wires having been connected to terminals of the incorrect polarity. The experiments in Dahlgren et al. (2014) also produced baseline noise levels that are 2-3 orders of magnitude higher than those reported in Scoville et al. (2015), calling into question the accuracy of the data reported in Dahlgren et al. (2014).
} 

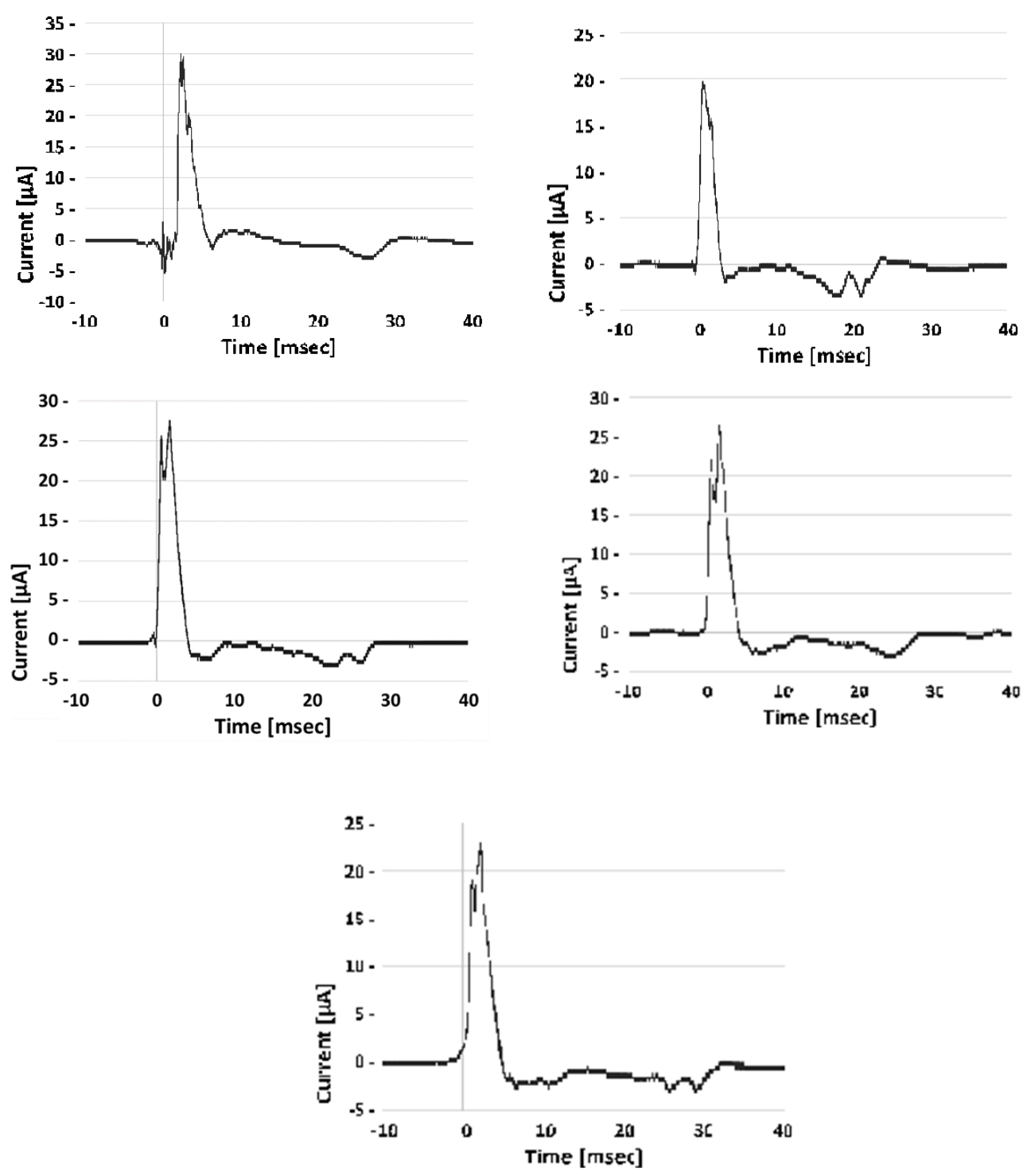

Figure 2. Current pulses observed after delivering a rapid impulse to gabbro samples.

conductors. These pulses also characteristically rise faster than they fall, a behavior that is also seen in most of the unipolar magnetic pulses shown in Fig. 1. This is also characteristic of the diffusion of charge carriers through semiconductors.

\section{Drift-diffusion semiconductor model}

The drift-diffusion equations are the most frequently used model for semiconductor physics, and perform well on scales greater than about $5 \times 10^{-7} \mathrm{~m}$ (Vasileska et al., 2008). They describe current in terms of charge carrier concentrations and an electrostatic field, and this determines the change in charge carrier concentrations via continuity of the current density.
The drift-diffusion equations are:

$$
\begin{aligned}
& \partial_{t} \boldsymbol{n}=-\boldsymbol{R}(\boldsymbol{n}, \boldsymbol{p})+\nabla \cdot\left(D_{n} \nabla \boldsymbol{n}-\mu_{n} \boldsymbol{n} \nabla \boldsymbol{V}\right) \\
& \partial_{t} \boldsymbol{p}=-\boldsymbol{R}(\boldsymbol{n}, \boldsymbol{p})+\nabla \cdot\left(D_{p} \nabla \boldsymbol{p}+\mu_{p} \boldsymbol{p} \nabla \boldsymbol{V}\right) \\
& \Delta \boldsymbol{V}=\frac{1}{\epsilon}(\boldsymbol{n}-\boldsymbol{p}-\boldsymbol{C}) .
\end{aligned}
$$

Here, $\boldsymbol{n}, \boldsymbol{p}, \boldsymbol{R}, \boldsymbol{V}$, and $\boldsymbol{C}$ are defined on a domain $\Omega \times(0, T)$, where $\Omega$ is a subset of a 3-D space on which these partial differential equations are to be solved. The functions $\boldsymbol{n}$ and $\boldsymbol{p}$ are concentrations of electron and hole charges, respectively, and $\boldsymbol{C}$ is the charge of any dopant ions that are present. $\boldsymbol{n}$ and $\boldsymbol{p}$ are constrained to be non-negative. $\boldsymbol{R}(\boldsymbol{n}, \boldsymbol{p})$ is the recombination and/or generation rate of electrons and holes. The third equation is Poisson's law of electrostatics whose solution describes the electric potential $\boldsymbol{V} . \epsilon$ is the electric 
permittivity. The constants $\mu_{n}$ and $\mu_{p}$ are the mobilities of electrons and holes, respectively, (not to be confused with the magnetic permeability $\mu$ or $\mu_{0}$ ) and $D_{n}$ and $D_{p}$ are the corresponding diffusion coefficients. In the particular instance of the model under consideration, $\mu_{n}$ and $D_{n}$ are approximately zero due to electrons becoming trapped in the valence band. The conductivity associated with a particular charge carrier is determined by the product of the number density of the charge carrier, the charge carried (one electronic charge, in this case), and its mobility.

\section{Coupling electromagnetism to drift-diffusion}

Maxwell's equations describe propagation at the speed of light, which is much faster than the charge carriers diffusing in a typical semiconductor. Rather than modeling propagation on two very different timescales, we make use of a quasistatic (magnetostatic) approximation (Jackson, 1999), assuming that currents do not alternate rapidly or approach the speed of light. Specifically, the Maxwell displacement current appearing in Ampere's law is assumed to be negligible: $c^{-2} \partial_{t} E \approx 0$. This assumption is justified for semiconductors, and is in fact implicit in the drift-diffusion model due to its use of Poisson's equation for the static electrical potential.

The electric current density $\boldsymbol{J}\left(\boldsymbol{x}^{\prime}\right)$ acts as the source of a magnetic field. It may be expressed as the sum of a drift term, involving the electric field, and a diffusive term, involving the concentration gradient. The rate of change of the concentration then becomes a continuity equation that is a function of current density. Explicitly separating the current and continuity equations facilitates coupling to the magnetic field. In this form, the current densities are:

$\boldsymbol{J}_{n}=D_{n} \nabla \boldsymbol{n}+\mu_{n} \boldsymbol{n} \nabla \boldsymbol{V}$

$\boldsymbol{J}_{p}=-D_{p} \nabla \boldsymbol{p}+\mu_{p} \boldsymbol{p} \nabla \boldsymbol{V}$.

The continuity equations that describe the change in electron and hole concentrations are then:

$\partial_{t} \boldsymbol{n}=-\boldsymbol{R}(\boldsymbol{n}, \boldsymbol{p})+\nabla \cdot \boldsymbol{J}_{n}$

$\partial_{t} \boldsymbol{p}=-\boldsymbol{R}(\boldsymbol{n}, \boldsymbol{p})+\nabla \cdot\left(-\boldsymbol{J}_{p}\right)$.

The current densities $\boldsymbol{J}_{n}$ and $\boldsymbol{J}_{p}$ are summed to obtain the total current density $\boldsymbol{J}$ that acts as a source for the magnetic field. In a magnetostatic approximation, the solution to the magnetic field on a domain may be efficiently computed by solving a set of Poisson equations for the magnetic vector potential. In this case, however, we calculate the field at an arbitrary point in space, which could be outside the domain.
We apply the Biot-Savart law to obtain the magnetic field at the point $\boldsymbol{x}$ :

$\boldsymbol{B}(\boldsymbol{x}, t)=\frac{\mu}{4 \pi} \int\left(\boldsymbol{J}_{p}\left(\boldsymbol{x}^{\prime}, t\right)+\boldsymbol{J}_{n}\left(\boldsymbol{x}^{\prime}, t\right)\right) \times \frac{\boldsymbol{x}-\boldsymbol{x}^{\prime}}{|\boldsymbol{x}-\boldsymbol{x}|^{\prime 3}} \mathrm{~d}^{3} \boldsymbol{x}^{\prime}$.

Here, $\left|\boldsymbol{x}-\boldsymbol{x}^{\prime}\right|$ is the magnitude of the vector from $\boldsymbol{x}$ to $\boldsymbol{x}^{\prime}$ and $\mu$ is the magnetic permeability. The velocities of the holes are not sufficiently large for the Lorentz force to be significantly influenced by magnetic fields so we do not consider the effect of the magnetic field on the charge carriers.

\section{Numerical solution}

The drift-diffusion equations are solved by expressing the partial differential equations as a system of ordinary differential equations for the time derivatives $\partial_{t} \boldsymbol{n}$ and $\partial_{t} \boldsymbol{p}$. A finitedifference approximation to this system is then integrated using a fourth-order Runge-Kutta scheme (RK4). Poisson's equation is solved separately at each timestep using successive over-relaxation (Golub and Van Loan, 1996) (SOR) with an adaptive relaxation parameter and open boundary conditions. For the other PDEs, the Dirichlet boundary conditions $n=0$ and $p=0$ are applied to the boundary of a grid of uniformly spaced points representing the $x, y$, and $z$ coordinates over which functions are evaluated. All spatial partial derivatives $\left(\partial_{x}, \partial_{y}, \partial_{z}, \nabla\right)$ of the current and continuity equations are approximated using a fourth-order central difference approximation.

At each timestep, the electric potential is determined by solving Poisson's equation, starting the SOR iteration with the electric potential from the previous timestep. Using the electric potential and the charge carrier concentrations, the components of the current vector fields $\boldsymbol{J}_{n}$ and $\boldsymbol{J}_{p}$ are evaluated. From $\boldsymbol{J}_{n}$ and $\boldsymbol{J}_{p}$, the continuity equations are integrated, yielding the concentrations of the charge carriers at the next timestep. The non-negativity constraint on $\boldsymbol{n}$ and $\boldsymbol{p}$ is enforced via truncation.

The magnetic field $\boldsymbol{B}$ is evaluated by applying a discretized Biot-Savart law to the currents. $\boldsymbol{B}$ is calculated at each timestep but since the result does not affect the dynamics, it may be evaluated at a single point.

\section{Results}

Since holes are mobile and electrons are immobile, diffusion separates the two species, creating an electric current that acts as an electromagnet. The boundary of a region of excess charge carriers behaves, essentially, like the $p$ - $n$ junction of a diode. Since only holes may flow out of this volume, the initial current diffusing across the boundary is unidirectional, corresponding to forward bias in the diode. However, after a delay period during which recombination reduces the diffusive current, the diode could switch to reverse bias, whereby 
the $p$ - $n$ junction capacitance generates a reverse recovery current. In the case of a recovery current, holes flow back into the source volume, producing a magnetic pulse that is opposite in polarity to the initial magnetic field.

We use the semiconductor model to calculate an example of a unipolar magnetic pulse. The electric permittivity and magnetic permeability are estimated based on the static properties of $\mathrm{MgO}$ (Batllo et al., 1991) $\left(\epsilon \approx 16.75 \epsilon_{0}\right.$ and $\left.\mu \approx \mu_{0}\right)$ and a temperature of $T=673.15 \mathrm{~K}$. Since electrons are trapped and immobile in broken peroxy bonds, $\mu_{n}$ and $D_{n}$ are set to 0 . The mobility and diffusion constant of holes were estimated based on experimental data from an experiment in which a rapid pressure impulse to the center of a gabbro tile injected holes that diffused and drifted away from their source, akin to a Haynes-Shockley experiment. The parameters used are $\mu_{p}=0.063 \mathrm{~m}^{2}(\mathrm{Vs})^{-1}$ and $D_{p}=8.5 \times 10^{-4} \mathrm{~m}^{2} \mathrm{~s}^{-1}$, roughly comparable to their values in pure undoped Silicon.

Charge generation is not explicitly considered in this calculation, and a pre-existing excess concentration of $10^{-5} \mathrm{C} \mathrm{m}^{-3}$ of both electrons and holes is an initial condition. These dissociated charges are initially present only at grid points inside a piriform teardrop surface of the form $(z / 4000)^{4}-(z / 4000)^{3}+(x / 2000)^{2}+(y / 1000)^{2}=0$. This surface geometry was chosen to avoid cancellation of fields due to spherical symmetry. The recombination rate is proportional to the product of electron and hole concentrations, $R=10^{22} \boldsymbol{n} \boldsymbol{p}$.

The attenuation of the magnetic field as it passes through the Earth is not considered, nor are the effects associated with the surface of the Earth. This is a reasonable approximation at extremely low frequencies. The fact that very slow (nearly DC) variations in the magnetic field can pass through the earth is the basis for magnetotelluric surveys. Ultra-low frequency radio waves are also used for through-the-earth radio communications in mine shafts due to the fact that lower frequency waves can penetrate the earth much more effectively than more conventional radio frequencies. This behavior is typical of most materials: low-frequency waves penetrate more effectively than high-frequency waves. The characteristic depth scale of penetration of an EM wave (the penetration depth or skin depth) (Jackson, 1999) is the reciprocal of the imaginary part of the wave vector, which, for a conducting medium, is:

$d=\frac{1}{\omega} \sqrt{\frac{\mu \epsilon}{2} \sqrt{1+\left(\frac{\sigma}{\epsilon \omega}\right)^{2}}-1} \approx \sqrt{\frac{2}{\mu \sigma \omega}}$.

Here, $\omega$ is the (angular) frequency, $\sigma$ is the conductivity, $\mu$ is the magnetic permeability, and $\epsilon$ is the electric permittivity.

Observed and calculated magnetic pulses are illustrated in Figs. 1 and 3 . Figure 3 shows the value of the $x$ component of the magnetic field as a function of time, evaluated $10 \mathrm{~km}$ directly above the center of the simulated volume. The amplitude, frequency, and shape of the pulse are sim-

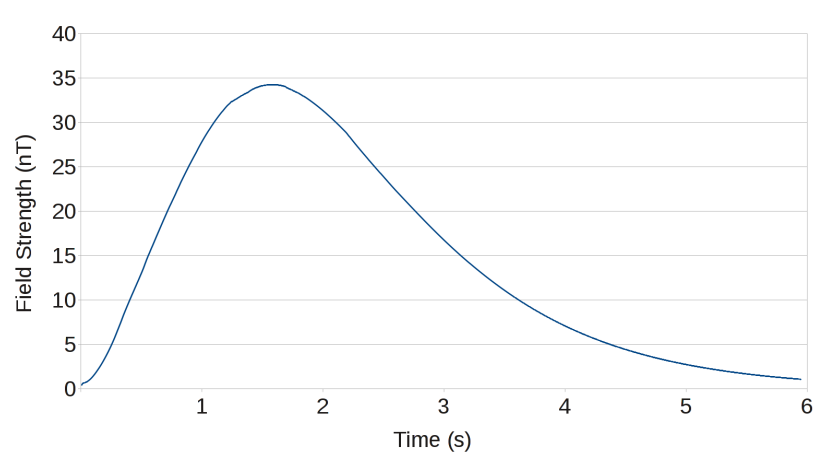

Figure 3. A calculated transient magnetic pulse, $10 \mathrm{~km}$ from the source volume.

ilar to pulses that have been observed before earthquakes. Figure 1 shows several magnetic pulses observed prior to an earthquake near Lima, Peru. These pulses were measured over a period of several days by a pair of magnetometers approximately $25 \mathrm{~km}$ away, and the locations of their sources were triangulated. The sources were clustered within a few kilometers of the epicenter of an earthquake that occurred 2 weeks after the onset of the pulses (Heraud et al., 2013). This analysis has been performed prior to several moderate earthquakes near Lima, with similar results.

The calculated pulse is unipolar and rises faster than it falls, like all but one of the pulses shown in Fig. 1. The duration of the calculated pulse is roughly $5 \mathrm{~s}$, falling within the range of $0.1-10 \mathrm{~s}$ for observed pulses. The intensity of the calculated pulse reaches $34 \mathrm{nT}$, whereas observed pulses have ranged from the noise floor (around $1 \mathrm{nT}$ ) to $200 \mathrm{nT}$ in intensity. Whereas the calculated pulse (which used a diffusion coefficient estimated using gabbro at room temperature) in Fig. 3 has a comparatively long duration and moderate intensity, the pulses shown in Fig. 1, have short duration $(\approx 200 \mathrm{~ms})$ but high intensity $(80-150 \mathrm{nT})$. Thus, in addition to having essentially the same form, the total energy of calculated and observed pulses are similar.

It is reasonable to expect that different rocks under different conditions will produce a variety of diffusion coefficient and mobilities, leading to a variety of pulse amplitudes and durations. If the charge carriers are not injected instantaneously, as we have modeled here, then different pulse shapes can result. For example, if charge injection is a continuous process rather than a rapid impulse, the rise of the pulse can be slower than its fall, as is the case for one of the pulses shown in Fig. 1.

\section{Conclusions}

In the model presented here, when a volume of rock enters a semiconducting state, e.g. from heat or stress, excess holes and electrons are injected. The mobile holes begin to dif- 
fuse out of the source volume, while electrons are trapped within the source volume and undergo recombination with the holes that have not diffused out. The flux of holes leaving the source effectively creates a $p-n$ diode: the source volume becomes an $n$ type semiconductor and the surrounding rock becomes $p$ type. A depletion region forms between the two layers of the $p-n$ junction and the $p-n$ double layer screens electric fields outside its immediate vicinity.

After charge injection, a diffusive current of holes flows as a result of the concentration gradient across the source boundary. This corresponds to a forward bias state of the diode, dominated by diffusion capacitance rather than junction capacitance. This current creates a transient magnetic field. As the hole concentration gradient decreases, the diffusive current and the magnetic field decay. After holes have diffused outward, creating $p$-type and $n$-type regions, a junction capacitance results from layers of positive and negative charge separated by a depletion region at the junction.

After a delay period, the diode may switch to a reverse bias state. In this case, electron-hole recombination consumes the holes remaining within the source volume, leaving mostly electrons inside. The junction capacitance causes a transient reverse recovery current. If the potential drop across the depletion region is sufficiently strong, reverse-bias electrical breakdown may occur as Coulomb attraction pulls holes back into the source volume.

It has been suggested (Freund, 2010) that circuit closure is necessary to produce electric currents and hence sources of magnetic fields. Although this may be a useful rule of thumb in everyday experience, it is not a law of physics, and the model developed here illustrates that continuous closed current loops (i.e. circuits) are not necessary to produce magnetic fields. The outflow current and subsequent return current associated with a large number of charge carriers is capable of producing significant magnetic fields, albeit transient, without formal circuit closure.

A distinctive form and the ability to pass through the earth at ultra-low frequencies make magnetic pulses potentially useful for the observation of pre-seismic shifts in the stress level of rocks that are otherwise inaccessible due to depth. Preliminary observations suggest that by triangulating the source of these magnetic pulses, the increased buildup of stress around future earthquake epicenters might be identified days or weeks in advance of seismicity.

In addition to unipolar pulses, other types of electromagnetic precursors or seismic electromagnetic phenomena might be predicted from semiconductor models. Oscillatory ULF fields, for example, have been observed immediately preceding earthquake activity (Bleier et al., 2009).

The positive hole semiconductor theory modeled here seeks to unify a wide range of electromagnetic phenomena associated with seismic activity. The direct coupling of semiconductor drift-diffusion currents and electromagnetism produces a model consistent with observations of preseismic magnetic pulses as well as laboratory experiments that rapidly load and unload rock samples. This agreement between theory, experiment, and field observations supports the hypothesis that pre-earthquake ULF activity might result from geophysical semiconductor processes.

Acknowledgements. The authors would like to thank Tom Bleier and Clark Dunson of QuakeFinder for many informative discussions. The authors would also like to thank Jiro Funamoto and Kim Johnson for their comments on the manuscript. This research was funded in part by NASA Earth Surface and Interior Grant NNX12AL71G (John LaBrecque).

Edited by: F. Masci

Reviewed by: two anonymous referees

\section{References}

Batllo, F., LeRoy, R. C., Parvin, K., Freund, F., and Freund, M. M.: Positive holes in magnesium oxide, Correlation between magnetic, electric, and dielectric anomalies, J. Applied Phys., 69, 6031-6033, 1991.

Bleier, T., Dunson, C., Maniscalco, M., Bryant, N., Bambery, R., and Freund, F.: Investigation of ULF magnetic pulsations, air conductivity changes, and infra red signatures associated with the 30 October Alum Rock M5.4 earthquake, Nat. Hazards Earth Syst. Sci., 9, 585-603, doi:10.5194/nhess-9-585-2009, 2009.

Bortnik, J., Bleier, T., Dunson, C., and Freund, F.: Estimating the seismotelluric current required for observable electromagnetic ground signals, Ann. Geophysics, 28, 1615-1624, 2010.

Campbell, W. H.: Natural magnetic disturbance fields, not precursors, preceding the Loma Prieta earthquake, J. Geophys. Res., 114, A05307, doi:10.1029/2008JA013932, 2009.

Dahlgren, R. P., Johnston, M. J., Vanderbilt, V. C., and Nakaba, R. N.: Comparison of the Stress-Stimulated Current of Dry and Fluid-Saturated Gabbro Samples, Bulletin of the Seismological Society of America, 2014.

Duba, A. and Shankland, T.: Free carbon \& electrical conductivity in the Earth's mantle, Geophys. Res. Lett., 9, 1271-1274, 1982.

Fraser-Smith, A., Bernardi, A., McGill, P., Helliwell, R., and Villard Jr., O.: Low-frequency magnetic field measurements near the epicenter of the Ms 7.1 Loma Prieta earthquake, Geophys. Res. Lett., 17, 1465-1468, 1990.

Fraser-Smith, A., McGill, P., and Bernardi, A.: Comment on "Natural magnetic disturbance fields, not precursors, preceding the Loma Prieta earthquake" by Wallace H. Campbell, J. Geophys. Res., 116, A08228, doi:10.1029/2010JA016379, 2011.

Freund, F.: Charge Generation and Propagation in Ingneous Rocks, J. Geodynam., 33, 543-570, 2002.

Freund, F.: On the electrical conductivity structure of the stable continental crust, J. Geodynam., 35, 353-388, 2003.

Freund, F.: Toward a unified solid state theory for pre-earthquake signals, Acta Geophys., 58, 719-766, 2010.

Freund, F., Masuda, M. M., and Freund, M. M.: Highly mobile oxygen hole-type charge carriers in fused silica, J. Mater. Res., 6, 1619-1622, 1991.

Freund, F., Takeuchi, A., and Lau, B.: Electric currents streaming out of stressed igneous rocks - A step towards understanding pre- 
earthquake low frequency EM emissions, Phys. Chem. Earth, 31, 389-396, 2006.

Golub, G. and Van Loan, C.: Matrix Computations, Johns Hopkins University Press, 3rd Edn., 1996.

Griscom, D.: Trapped-electron centers in silica, J. Non-Cryst. Solids, 357, 1945-1962, 2011.

Harris, R.: Nonclassical Physics: Beyond Newton's View, Addison Wesley, 1998.

Heraud, J., Centa, V. A., Bleier, T., and Dunson, C.: Determining future epicenters by triangulation of magnetometer pulses in Peru, in: AGU Fall Meeting, NH014, 2013.

Jackson, J.: Classical Electrodynamics, John Wiley and Sons, 3rd Edn., 1999.

King, B. and Freund, F.: Surface charges and subsurface spacecharge distribution in magnesium oxides containing dissolved traces of water, Phys. Rev. B, 29, 5814-5824, 1984.

Moore, G.: Magnetic disturbances preceding the 1964 Alaska earthquake, Nature, 203, 508-509, 1964.

Revil, A., Naudet, V., Nouzaret, J., and Pessel, M.: Principles of electrography applied to self-potential electrokinetic sources and hydrogeological applications, Water Resour. Res., 39, 1114, doi:10.1029/2001WR000916, 2003.

Ricci, D., Pacchioni, G., Szymanski, M., Shluger, A., and Stoneham, A.: Modeling disorder in amorphous silica with embedded clusters: the peroxy bridge defect center, Phys. Rev. B, 64, 224104, doi:10.1103/PhysRevB.64.224104, 2001.

Scoville, J., Sornette, J., and Freund, F.: Paradox of peroxy defects and positive holes in rocks Part II: Outflow of electric currents from stressed rocks, J. Asian Earth Sci., in press, doi:10.1016/j.jseaes.2015.04.016, 2015.
Selway, K.: On the Causes of Electrical Conductivity Anomalies in Tectonically Stable Lithosphere, Surveys in Geophysics, 35, 219-257, doi:10.1007/s10712-013-9235-1, 2014.

Shluger, A., Heifets, E., Gale, J., and Catlow, C.: Theoretical simulation of localized holes in MgO, J. Phys.: Condens. Matter, 4, 5711-5722, 1992.

Shockley, W., Pearson, G., and Haynes, J.: Hole Injection in Germanium - Quantitative Studies and Filamentary Transistors, Bell Syst. Techn. J., 28, 344-366, 1949.

Shockley, W.: "Transistor Technology Evokes New Physics”, Nobel lecture, Nobel Lectures, Physics 1942-1962, 1964.

Thériault, R., St-Laurent, F., Freund, F., and Derr, J.: Prevalence of Earthquake Lights Associated with Rift Environments, Seismol. Res. Lett., 85, 159-178, 2014.

Thomas, J. N., Love, J. J., and Johnston, M.: On the reported magnetic precursor of the 1989 Loma Prieta earthquakes, Phys. Earth Planet. Int., 173, 207-215, 2009.

Unsworth, M., Egbert, G., and Booker, J.: High-resolution electromagnetic imaging of the San Andreas fault in Central California, J. Geophys. Res., 104, 1131-1150, 1999.

Vallianatos, F., Triantis, D., Tzanis, A., Anastasiadis, C., and Stavrakas, I.: Electric earthquake precursors: from laboratory results to field observations, Physics and Chemistry of the Earth, Parts A/B/C, 29, 339-351, 2004.

Varotsos, P.: The Physics of Seismic Electric Signals, TerraPub, 2005.

Vasileska, D., Mamaluy, D., Khan, H., Raleva, K., and Goodnick, S.: Semiconductor Device Modeling, J. Comput. Theor. Nanos., 5, 999-1030, 2008. 